\title{
Références bibliographiques du dossier «qualité, équité et diversité dans le préscolaire »
}

Cécile de Bouttemont

\section{(2) OpenEdition \\ 1 Journals}

Édition électronique

URL : http://journals.openedition.org/ries/919

DOI : 10.4000/ries.919

ISSN : 2261-4265

Éditeur

Centre international d'études pédagogiques

Édition imprimée

Date de publication : 1 avril 2010

Pagination : 141-150

ISSN : 1254-4590

\section{Référence électronique}

Cécile de Bouttemont, « Références bibliographiques du dossier « qualité, équité et diversité dans le préscolaire » », Revue internationale d'éducation de Sèvres [En ligne], 53 | avril 2010, mis en ligne le 01 avril 2011, consulté le 01 mai 2019. URL : http://journals.openedition.org/ries/919 ; DOI : 10.4000/ ries.919

(c) Tous droits réservés 


\section{Références \\ bibliographiques}

\section{Cécile de Bouttemont}

De nouvelles problématiques générées par l'évolution de notre mode de vie orientent, pour le monde de la petite enfance, la réflexion vers les questions d'équité et d'accueil de la diversité au niveau du préscolaire. Afin de mieux situer l'approche et la singularité de la thématique de ce numéro, cette bibliographie propose une sélection de références privilégiant une approche comparée de la question. Elles sont classées sous quatre thèmes :

- les perspectives directement liées à cette évolution et les nouveaux enjeux qui en découlent ; ces ouvrages de recherche et de réflexion sont suivis d'un état des lieux sur le préscolaire, fondé sur divers rapports internationaux ;

- les nouvelles orientations et les politiques mises en œuvre dans quelques pays pour favoriser l'accueil et la reconnaissance de la diversité ;

- les pratiques d'enseignement et les pédagogies mises en place qui se distinguent les unes des autres par le contexte et les valeurs culturelles de chaque pays ;

- les acteurs du préscolaire, en particulier parents et professionnels, qui vivent une évolution de leur rôle traditionnel vers de nouvelles formes d'implication.

La plupart de ces publications sont postérieures à 2007 et reflètent l'état de la recherche dans ce domaine d'étude. Certaines sont consultables au centre de ressources et d'ingénierie documentaires du CIEP ou accessibles en ligne.

Bibliographie arrêtée le 11 mars 2010.

\section{NOUVELleS APPROCHES, NOUVEAUX ENJEUX}

BROUGERE, G., VANDENBROECK M., Repenser l'éducation des jeunes enfants, PIE Peter Lang/Bruxelles, 2007, 288 p.

Cet ouvrage rassemble les analyses de nombreux auteurs dans le domaine de la recherche et des politiques de la petite enfance. La première partie, développée par des auteurs anglophones, présente des perspectives critiques appliquées aux contextes de chaque pays. Les contributeurs francophones de la seconde partie renouvellent la réflexion sur l'éducation préscolaire.

\section{DAHLBERG G., MOSS P., PENCE A., Beyond Quality in Early Childhood Education} and Care: Languages and Evaluation, Routledge/London 2007, 232 p.

Cette deuxième édition traite des questions de la petite enfance par une approche pluridisciplinaire : philosophie, éthique, sociologie de l'enfance et sciences politiques sont abordées. Les auteurs, qui s'appuient sur des travaux effectués au Canada, en Suède et en Italie, analysent le concept de qualité, sa définition, sa mesure. Ils mettent en garde contre la tendance à réduire ce concept à des aspects techniques qui en masquent la profondeur philosophique.

GENEIX N., CHARTIER L. dir., Petite enfance : enjeux éducatifs de 0 à 6 ans, ESF/Paris, 2007, 171 p.

Cet ouvrage propose une vision d'ensemble de la petite enfance et des nouveaux enjeux liés à son évolution. Modes de garde, apprentissage des enfants et partenariat sont analysés en vue d'améliorer pour chacun de ces thèmes qualité et équité. Enfin, les auteurs réfléchissent sur la prise en compte du préscolaire au niveau politique. 
HAGGLUND S, PRAMLING SAMUELSSON I., " Early Childhood Education and Learning for Sustanable Development and Citizenship », International Journal of Early Childhood, septembre 2009, vol 41, $n^{\circ} 2$, p. 49-63.

Dans cet article, les auteurs mettent l'accent sur l'importance du rôle de l'éducation de la petite enfance dans le développement durable. Pour illustrer leur propos, les auteurs prennent appui sur les récents changements dans la politique préscolaire suédoise, en particulier la pédagogie à l'école maternelle.

\section{MOSS P., Early Childhood education: Markets and democratic Experimentalism,} Institut of education/London, 2008, 45 p., http://www.bertelsmann-stiftung.de/ bst/de/media/xcms_bst_dms_24015__2.pdf/.

L'auteur décrit et compare deux modèles d'éducation de la petite enfance, l'un fondé sur la relation acheteur-fournisseur, l'autre sur des relations de dialogue et de créativité entre citoyens. Les deux modèles sont analysés sous l'angle de leurs objectifs, de leurs concepts et de leurs valeurs et sont comparés quant à leurs implications sur la structuration du système préscolaire : accès, offre de services, management, financement et personnel.

\section{PENN H., dir., Early Childhood Services. Theory, policy and practice, Open University Press/Buckingham, 2000, 201 p.}

Les auteurs de cet ouvrage explorent les relations entre théorie, politique et pratique dans les services de la petite enfance. En provenance de toute l'Europe les contributions analysent les atouts et les limites des différentes pratiques et suggèrent différentes pistes d'amélioration.

\section{RINALDI C., In dialogue with Reggio Emilia, Routledge/London, 2006, 240 p.}

Ce livre propose une série d'articles, de conférences et d'interviews de 1994 à nos jours. Parmi les thèmes abordés, la singularité du système préscolaire mis en place à Reggio Emilia. L'art et la créativité dans l'éducation préscolaire sont mis en avant.

\section{STAPLES NEW R., COCHRAN M., Early childhood Education: an international encyclopedia, Greenwood Publishing Group/Boston, 2007, 1448 p.}

Cette encyclopédie en quatre volumes rassemble des articles théoriques, politiques et didactiques sur nombre de sujets et controverses de l'éducation préscolaire. Les trois premiers volumes contiennent plus de trois cents entrées sur le développement de la petite enfance et l'éducation aux États-Unis et couvrent les grandes questions historiques et contemporaines, les recherches actuelles, et une variété de perspectives théoriques et politiques. Toutes les entrées se concluent par une liste des ouvrages cités ainsi que des lectures recommandées. En outre, les entrées sont rassemblées dans une section qui les regroupe par thèmes. Le quatrième volume contient plus d'une centaine d'entrées sur la structure et les politiques d'éducation de la petite enfance dans dix pays, couvrant tous les continents en dehors de l'Amérique du Nord. Les pays ont été sélectionnés pour mettre en valeur l'éducation de la petite enfance dans un large éventail de sociétés.

\section{VANDENBROECK M., Éduquer nos enfants à la diversité sociale, culturelle,} ethnique, familiale, Erès/Ramonville Sainte Anne, 2005, 240 p.

Selon l'auteur, l'accueil de la diversité constitue l'un des enjeux majeurs actuels dans l'éducation à la petite enfance. Il propose ainsi des orientations pédagogiques concrètes en s'inspirant de la littérature belge, française anglaise et américaine. Il développe des pistes de réflexion pour une pédagogie de la rencontre. 
VANDENBROECK M., "Beyond anti-bias education: changing conceptions of diversity and equity in European early childhood education ", European Early Childhood Education Research Journal, $n^{\circ}$ 15, vol. 1, 2007, p. 21-35.

Cet article traite plus particulièrement de l'évolution des discours sur la diversité et l'équité dans l'éducation de la petite enfance. Selon l'auteur, certains concepts qui sous-tendent ces questions peuvent être interrogés. L'évolution de la société implique de nouvelles recherches sur la politique et la pratique concernant l'accueil de la diversité dans l'éducation à la petite enfance.

\title{
LE PRÉSCOLAIRE, UN ÉTAT DES LIEUX : RAPPORTS INTERNATIONAUX
}

\author{
BENNETT J., MOSS P., Working for inclusion: an overview of European Union of \\ early years services and their workforce, 2010, 67 p., http://www.childreninscotland. \\ org.uk/docs/WFI_Res_report_Jan10.pdf/. \\ Ce rapport fait partie du programme européen sur l'éducation inclusive, Working for inclusion, \\ qui examine la façon dont l'amélioration des compétences et des qualifications des professionnels \\ de la petite enfance permet de réduire les inégalités et la pauvreté. Des recherches sont menées \\ dans une dizaine de pays : Royaume-Uni, Pologne, Norvège, Italie, Slovénie, France, Danemark, \\ Portugal, Suède et Hongrie. Les résultats doivent contribuer à l'élaboration de politiques au \\ niveau de l'Union européenne.
}

EACEA (Agence exécutive Éducation audiovisuel et culture), L'éducation et l'accueil des jeunes enfants en Europe : réduire les inégalités sociales et culturelles à l'école, Eurydice/Bruxelles, 2009, 194 p., http://eacea.ec.europa.eu/.

Le rapport examine les données transnationales et les politiques nationales de trente pays d'Europe relatives à l'éducation et l'accueil des jeunes enfants qui n'ont pas atteint l'âge de la scolarité obligatoire. Il est organisé en trois sections une vue d'ensemble de la littérature scientifique sur l'impact des mesures d'éducation et d'accueil des jeunes enfants ; une présentation des données statistiques en matière démographique ; une analyse comparative des mesures politiques menées dans ces pays pour l'organisation et le financement des services ainsi que pour la formation du personnel. L'accent est mis dans cette étude sur les politiques axées sur les enfants à risque.

CIDREE (Consortium of institutions for Development and Research in Education in Europe), The education of 4-to-8 years old: redesigning school entrance phase, CIDREE Administration/Brussels, 2007, 144 p.

Ce rapport rend compte de la diversité de la scolarisation des enfants âgés de quatre à huit ans dans huit pays européens. Les politiques mises en ouvre, les curriculums et les pratiques d'enseignement sont décrites, l'enjeu étant de permettre à chaque enfant d'être inclus dans le système préscolaire.

KORPI B., The politics of preschool: intentions and decisions underlying the emergence and growth of the Swedish pre-school, Ministère de l'Éducation suédois, 2007, 88 p., http://www.see-educoop.net/education_in/pdf/swedish-preschool.pdf

Ce rapport propose une vue d'ensemble de la politique mise en place en Suède pour favoriser le développement de l'éducation préscolaire. Il s'attache plus particulièrement aux activités mêlant soins et pédagogie sur les enfants de 1 à 6 ans. 
OCDE (Organisation de coopération et de développement économiques), Petite enfance grands défis II : éducation et structures d'accueil, OCDE/Paris, 2007, $504 p$.

Douze pays de l'OCDE, essentiellement européens, participent à l'examen thématique de la politique d'éducation et d'accueil des jeunes enfants n'ayant pas encore atteint l'âge de l'obligation scolaire lancé par le Comité de l'éducation de l'OCDE en 1998. Des données d'ensemble ont été recueillies, permettant une analyse comparative tout en dégageant un certain nombre de stratégies d'action.

UNESCO/CONSEIL DE L'EUROPE, Éducation des enfants roms en Europe. Vers une éducation de qualité pour les enfants roms : la transition entre le préscolaire et le primaire, Paris/UNESO, 2007, 43 p., http://www.coe.int/t/dg4/education/ roma/Source/CDE-UNESCO_FR.pdf/.

Après avoir fourni un aperçu de la situation, les contributeurs de ce rapport proposent des politiques et des pratiques qui semblent prometteuses pour favoriser l'accès à l'enseignement préscolaire puis une transition en douceur vers l'enseignement primaire. Le rôle à jouer par les familles et la communauté rom est également abordé.

UNICEF, La transition en cours dans la garde et l'éducation de l'enfant: tableau de classement des services de garde et d'éducation des jeunes enfants dans les pays économiquement avancés, Florence/Centre de recherche Innocenti, 2008, 40 p., http://www.lefuret.org/images/UNICEF.pdf

Dans ce bilan, dix points de référence sont proposés comme une première tentative d'établir un tronc commun minimal de normes en matière de services à la petite enfance. Les conclusions de ce rapport plaident pour un engagement massif des pays et pour un élargissement du débat et des investissements en matière d'accessibilité, de qualité et d'équité.

\section{LES POLITIQUES DE LA PETITE ENFANCE}

"Dossier : scolariser la petite enfance?", Perspectives, décembre 2004, n 132, 140 p., http://www.ibe.unesco.org/fileadmin/user_upload/archive/publications/Prospects/ ProspectsPdf/132/132-f.pdf/.

Composé de huit articles rédigés par des spécialistes de différents pays, ce dossier fait le point sur la situation de la petite enfance en rapport avec la question de l'âge de la préscolarisation. Il fait suite à un colloque international ayant réuni à Genève en 2003 chercheurs, administrateurs et professionnels. Malgré de fortes différences d'une région à l'autre, il existe une préoccupation commune fondée sur l'importance croissante de l'éducation dans le développement économique et social de tous les pays.

EINARSDOTTIR J., WAGNER J. T. dir., Nordic Childhoods and Early Education: Philosophy, Research, Policy and practice in Denmark, Finland Iceland, Norway and Sweden, Information Age Publishing/Greenwich, 2005, 324 p.

Politiques, pratiques et réflexions sur l'éducation à la petite enfance sont analysées dans une perspective comparée par de nombreux chercheurs et éducateurs. Sont abordées les réformes de la formation des enseignants, la question de l'apprentissage et la transition du préscolaire à l'école primaire. 
KAGAN S. L., "American early childhood education: Preventing or perpetuating inequity? ", Research Review, $n^{\circ}$ 3, 2009, 31 p., http://www.equitycampaign.org/ i/a/document/9833_EquityMatters_Kagan_Final.pdf/.

Selon l'auteur, le système préscolaire américain est fortement inégalitaire alors qu'il aurait les moyens de diminuer fortement ces disparités. Face à ce paradoxe, l'auteur analyse la politique du préscolaire, les différentes formes d'inégalités (qualité de l'éducation, personnel enseignant, infrastructures, etc.) et formule des recommandations pour réformer l'enseignement préscolaire.

\section{KAMERMAN S. dir., Early Childhood Education and Care: international perspectives, Columbia University Press/Columbia, 2001, 332 p.}

Dans cet ouvrage, l'auteur oppose les perspectives américaine et européenne sur l'éducation de la petite enfance. Il décrit les principales politiques européennes d'intervention précoce et leurs fondements politiques et sociaux. Un résumé des politiques mises en place dans six pays européens (Danemark, France, Italie, Finlande, Suède et Angleterre) est proposé.

\section{"Les politiques éducatives de la petite enfance ", Politiques d'éducation et de} formation, 2002/3, $n^{\circ}$ 6, $105 p$.

Les nombreux articles de ce numéro abordent différents aspects liés aux politiques de la petite enfance. Après l'analyse de ce que recouvre le terme même de politique de la petite enfance, les auteurs analysent la notion de qualité, le cadre pédagogique, les systèmes intégrés et les coordonnateurs de la petite enfance dans une perspective comparée. La dimension sanitaire liée à cette politique est abordée dans le dernier article.

NEUMAN M., "Governance of early childhood education and care: recent developments in OECD countries ", Early Years, vol. 25, $n^{\circ} 2$; juillet 2005, p. 129-141, http://www.informaworld.com/smpp/title $\sim \mathrm{db}=\mathrm{all} \sim$ content $=\mathrm{t} 713422238 \sim \mathrm{tab}=\mathrm{is}$ sueslist $\sim$ branches $=25-\mathrm{v} 25 /$.

Cette étude comparative qui porte sur douze pays de l'OCDE montre différentes approches en matière de politique préscolaire. Elle met en avant le rôle du gouvernement central par rapport aux autorités locales, l'implication des parents, et des professionnels de la petite enfance. Elle appelle enfin à des recherches empiriques concernant les effets de la gouvernance sur la nature des politiques et des programmes du préscolaire.

PAPON Monique, MARTIN Pierre, La scolarisation des jeunes enfants, Rapport d'information du sénat, $n^{\circ}$ 47, octobre 2008, $92 p$.

Dans ce rapport, les auteurs posent la question du bien-fondé de l'entrée des moins de 3 ans dans un cadre scolaire, en France. Partisans et détracteurs de la scolarisation s'affrontent sur deux logiques : une scolarisation précoce comme facteur de réduction des inégalités sociales et de prévention de l'échec scolaire ou une inadaptation de l'institution scolaire à la prise en compte du développement et du bien-être du jeune enfant. Il passe en revue la politique de scolarisation dans le contexte culturel français, aborde la question de la pédagogie et finit par une série de préconisations.

\section{STAPLES NEW R., LEVINE R. A., Anthropology and Child Development: A Cross-Cultural Reader, Blackwell Publishers/Oxford, 2008, 336 p. \\ Les articles rassemblés dans cet ouvrage analysent de manière comparative la politique préscolaire mise en place sur plusieurs continents en fonction du développement de l'enfant. Le contexte culturel, par ses traditions et ses valeurs, implique une vision politique qui se distingue d'un pays à l'autre.}


TAKEUCHI M., SCOTT R., dir., New directions for Early Childhood Education and Care in the 21st Century: International perspectives, Quam Martin Press, 2006, $288 p$.

Cet ouvrage analyse l'éducation de la petite enfance et ses perspectives liées à l'évolution de la société. Les auteurs se penchent plus particulièrement sur la structure de programmes de la petite enfance et l'âge de l'entrée dans le préscolaire. Plusieurs chapitres explorent l'éducation préscolaire dans les pays suivants : les États-Unis, le Japon et la Grande-Bretagne.

\title{
Pratiques d'enseignement ET VALEURS CULTURELLES
}

\author{
BOLSTERLI M., dir., MAULINI O., dir., L'entrée dans l'école : rapport au savoir \\ et premiers apprentissages, De Boeck/Bruxelles, 2007, 167 p.
}

Cet ouvrage traite des premières années de scolarité dans trois pays européens : la Belgique, la France et la Suisse. La première partie analyse les pratiques pédagogiques des enseignants et les conceptions du savoir dont elles sont l'incarnation. La deuxième partie étudie le rapport entre les pratiques observées et ce que peuvent en apprendre les élèves ; elle propose des outils pour mieux comprendre comment les enfants et leurs familles abordent le savoir et la culture scolaires.

\section{BOUVE C., RAYNA S., MOISSET P., Pour un accueil de qualité de la petite} enfance : Quel curriculum ?, Erès/Toulouse, 2009, 412 p.

Cet ouvrage rassemble les contributions de professionnels de l'accueil, de responsables municipaux et de chercheurs et formateurs. Après avoir défini la notion de curriculum et ses enjeux, les auteurs présentent des expériences novatrices en Italie et en Belgique. Les parties suivantes sont consacrées au curriculum, à sa construction en tant que processus démocratique et participatif. La coéducation est abordée à partir d'une pluralité d'expériences, le jeu et l'accueil de la diversité sont explorés dans des contextes différents.

BROUGERE G., GUENIF-SOUILAMAS N., RAYNA S., "École maternelle in France: a cross-cultural perspective ", European Early Childhood Education Research Journal, vol. 16, $n^{\circ}$ 3, septembre 2008, p. 371-384.

Cet article présente une analyse des discours croisés d'enseignants du préscolaire et de parents (migrants et non migrants) sur les enfants de migrants à l'école maternelle. Recueillis en France, ces discours sont confrontés aux voix venues des États-Unis. Les thèmes abordent la question du jeu, des apprentissages, et de la langue. Cette confrontation laisse apparaître différents modèles normatifs et les valeurs culturelles mobilisées par chacun des protagonistes ainsi que leur traduction au quotidien.

\footnotetext{
BROUGERE G., RAYNA S., Culture, enfance et éducation préscolaire, Unesco/ Paris, 1998, 268 p.

Cet ouvrage rassemble les actes d'un séminaire portant sur la dimension culturelle de l'éducation préscolaire. Il comporte treize contributions de spécialistes venus de quatre continents et travaillant dans des champs disciplinaires différents. Organisé en plusieurs parties, il introduit la problématique de l'ancrage culturel et se consacre aux approches comparatives des pratiques éducatives à l'école maternelle aux États-Unis, en Chine et au Japon. Il se poursuit avec l'incidence des représentations de l'enfant sur l'éducation préscolaire, les valeurs culturelles et sociales, les solidarités familiales en prenant des exemples au Mali et en France.
} 
FAVARO G., MANTOVANI S., MUSATTI T., Une crèche pour apprendre à vivre ensemble, Erès/Toulouse, 2008, 264 p.

Rédigé à partir d'une recherche sur l'accueil des enfants de migrants dans les structures de la petite enfance de trois villes italiennes, Rome, Milan et Pérouse, cet ouvrage présente un aspect des différentes cultures de l'enfance, par l'intermédiaire des paroles de mères immigrées et d'éducatrices travaillant dans des lieux multiculturels. Il invite les professionnels de la petite enfance et toute personne confrontée aux problématiques de la diversité et de l'intégration, à repenser l'accueil des familles d'origine étrangère.

\section{GANDINI L., POPE EDWARDS C., Bambini. The Italian Approach to Infant/} Toddler Care, New York/Teachers College Press, 2001, 237 p.

Cet ouvrage analyse l'approche originale sur l'éducation des jeunes enfants mise en place dans quatre villes italiennes. Combinant soins et éducation, cette approche tente de développer l'aspect affectif et cognitif et prend en compte la relation entre parents et petits.

\section{PASCAL C., BERTRAM T., Early years education: an international perspective,} London/Qualification and curriculum authority, NFER-QCA/London, 2002, 69 p. http://www.inca.org.uk/pdf/early_years.pdf

L'organisme anglais NFER, qui a pour mission l'évaluation des curricula et des qualifications, a mené une étude dans vingt pays sur les curricula dans l'éducation préscolaire. Cette étude porte en particulier sur le contenu des programmes, très hétérogènes dans leur prescription et dans leurs lignes directrices, la pédagogie, les qualifications du personnel, la transition avec l'enseignement primaire, l'assurance qualité et l'évaluation.

PRAMLING SAMUELSSON I., SHERIDAN S., WILLIAMS P., " Five preschool curricula - comparative perspective », International Journal of Early Childhood, mars 2006, vol. 36, $n^{\circ} 1$, p. 11-30.

Cette étude compare les différents contextes sociaux et culturels dans lesquels se déroulent les apprentissages des jeunes enfants au niveau préscolaire. L'objectif de cet article est d'analyser les différences et les similitudes existant entre les programmes d'éducation et de discuter leur niveau de qualité du point de vue pédagogique. Les résultats de l'analyse montrent que les cinq programmes sont de haute qualité par rapport à la culture de chacun des pays dont ils sont issus.

\section{PRAMLING SAMUELSSON I., FLEER M., Play and learning in early childhood} settings international perspectives, Springer, 2008, 195 p.

Cet ouvrage est une étude sous l'angle historique et culturel du jeu des enfants de la naissance à 2 ans. Des chercheurs de Nouvelle-Zélande, d'Australie, du Chili, de Hong-Kong, de Chine continentale, du Japon, de la Suède et des États-Unis présentent leurs enquêtes sur les activités ludiques des nourrissons et des tout-petits dans leurs différents milieux culturels.

\section{RAYNA S., BROUGERE G., coord., Traditions et innovations dans l'éducation préscolaire : perspectives internationales, INRP/Paris, 2000, 511 p.}

Cet ouvrage est le résultat de la réflexion menée lors d'un séminaire organisé par le CRESASINRP et le GREC (Université Paris 13) de 1996 à 1998. Il analyse les politiques, les pratiques d'enseignement de l'éducation préscolaire. Il rassemble, dans une perspective comparative mettant en valeur l'ancrage culturel, les travaux de spécialistes de treize pays répartis sur quatre continents. 
TRINQUIER M.-P., ZERBATO-POUDOU M.-T., " Le préscolaire en question : Questions sur les pratiques ", Les dossiers des sciences de l'éducation, 2002, $n^{\circ} 7,120 p$.

Ce dossier pose d'emblée la question des formes préscolaires et de leurs manifestations pragmatiques. Contrairement à d'autres pays, l'école maternelle française valorise les pratiques de type scolaire. Les enjeux de l'éducation préscolaire sont abordés à travers la question de la qualification et de la formation des personnels. Les pratiques des enseignants sont analysées dans différents contextes culturels sous l'angle didactique.

\section{ACteurs de la Petite enfance}

BEAUDELOT O., RAYNA S., MAYER S. MUSATTI T., « A comparative analysis of the function of coordination of early childhood education and care in France and Italy », Early Years Education, juin 2003, $n^{\circ}$ 11, p. 105-116.

Cette étude fournit une analyse comparative de la fonction de coordination des services de la petite enfance en France et en Italie à partir de deux enquêtes menées dans des municipalités sur le statut, la formation et les tâches des coordinateurs de la petite enfance. Les implications de cette fonction de coordination dans la promotion de la qualité des soins et de l'éducation dans les services de la petite enfance sont discutées.

BLANC M.-C., BONNABESSE L., "L'évolution de la place des parents dans les établissements d'accueil de jeunes enfants ", Spirale, avril 2008, n 48, p. 115-129.

Les auteurs abordent cette question selon deux perspectives : l'institutionnalisation progressive de la place des parents et le positionnement des professionnels dans leur relation aux parents.

\section{BOUVE C., Les crèches collectives : usagers et représentations sociales. Contri- bution à une sociologie de la petite enfance. L'Harmattan/Paris, 2001, 310 p.}

Entre l'histoire et la sociologie, cet ouvrage propose, dans un premier temps, de questionner le sens de l'évolution socioprofessionnelle des usagers de la crèche. Dans un second temps, l'analyse sociologique, permet d'appréhender les motivations qui conduisent à choisir la crèche collective comme mode de garde pour son enfant et les relations qui se construisent entre familles et institutions.

\section{BROUGERE G., RAYNA S., coord. Accueillir et éduquer la petite enfance. Les relations entre parents et professionnels, INRP/Paris, 2005, $186 \mathrm{p}$.}

Cet ouvrage analyse les relations entre parents et professionnels de la petite enfance. Issu d'un séminaire organisé à l'INRP, les regards croisés de chercheurs français et étrangers apportent des réponses sur certains aspects jusqu'ici peu explorés par la recherche. L'observation des pratiques et l'analyse du discours des parents et des professionnels permettent de faire ressortir plusieurs types de relations entre familles et institutions, et plusieurs modalités de communication.

FRAISSE L., LHUILLIER V., PETRELLA F., "L'accueil des jeunes enfants en Europe : vers des formes de gouvernance multilatérale et intégrée ", Revue française de socio-économie, février 2008, $n^{\circ}$ 2, p. 141-160.

Cet article analyse les mutations du secteur de la petite enfance sous l'angle des interactions entre acteurs publics, acteurs associatifs et privés. Les enjeux qui découlent de l'évolution des modes de gestion modifient le rôle de l'État et impliquent une diversité d'acteurs dont il convient de déterminer le rôle et la place. 


\section{JESU Frédéric, Co-éduquer. Pour un développement social durable, Dunod/Paris, 2004, $189 p$.}

Cet ouvrage met en avant l'importance de la participation de tous les acteurs concernés par l'éducation des jeunes enfants : parents, professionnels, bénévoles et enfants. Il plaide pour le développement d'initiatives globales. Il s'adresse aux décideurs politiques et aux acteurs professionnels et associatifs impliqués dans le secteur de l'éducation des jeunes enfants.

KARLSSSON LOHMANDER M., "The Fading of a Teaching Profession ", Early Years, mars 2004, vol. 24, $n^{\circ} 1$, p. 23-34.

Après un historique de la mise en place de l'éducation des jeunes enfants en Suède au fil des réformes, l'auteure analyse les conséquences de ces changements pour les professionnels de la petite enfance.

\section{KHERROUBI M., dir., Des parents dans l'école, Erès/Toulouse, 2008, 224 p.}

Ce livre présente le résultat de recherches menées dans dix-huit établissements primaires afin d'analyser les différentes modalités de coopérations entre parent et établissement. On y découvre la façon dont s'organise quotidiennement cette relation dans des écoles aux caractéristiques contrastées, les enjeux et les facteurs de cette coopération et ses conséquences sur la scolarité des enfants.

LESUR E., «De l'espace professionnel à l'espace éducatif : une analyse comparative des pratiques de deux professionnels de la petite enfance ", Pensée plurielle, février 2008, $n^{\circ} 18$, p. 139-159.

Le choix du mode de garde pour les enfants d'âge préscolaire est loin de faire l'objet d'un consensus, si les enseignants accueillent environ un tiers des deux ans à l'école maternelle, des modes de garde alternatifs existent. Dans ces structures, d'autres professionnels posent un regard différent sur les mêmes enfants et proposent un autre type d'accueil. Cet article se propose de comparer les pratiques de ces deux types de professionnels de la petite enfance : l'enseignante et l'éducatrice de jeunes enfants.

PEETERS J., The construction of a new profession, SWP/Amsterdam, 2008, $176 \mathrm{p}$.

Les professions dans le secteur de l'enfance sont en cours de réforme dans de nombreux pays. Les exemples de cet ouvrage, issus du Danemark et de Nouvelle-Zélande, illustrent la possibilité de construire un professionnalisme accessible à tous dans l'éducation de la petite enfance.

\section{RAYNA S., RUBIO M.-N., SCHEU H., coord., La coéducation en question, Erès/} Toulouse, 2010.

Les chapitres de cet ouvrage analysent la notion, les modalités et les enjeux de la coéducation sous l'angle du partenariat parent-professionnel dans le domaine de l'éducation des jeunes enfants. Ils proposent des réflexions et des mises en pratique à l'école maternelle, la crèche et les lieux d'accueil enfants - parents de plusieurs pays.

\section{SAADA E. H., DUCRET J.-J., Représentations des pratiques éducatives chez les} professionnels de la petite enfance, Service de Recherche en éducation/Genève, 84 p., http://www.geneve.ch/sred/publications/docsred/2009/RepPraticEducative.pdf

Cette étude sur les représentations croisées du métier et des pratiques pédagogiques chez les éducatrices et chez les enseignantes. Elle examine les priorités dans les objectifs éducatifs visés, la place accordée à des activités d'apprentissage, les contenus cognitifs considérés et le passage des enfants entre les institutions de la petite enfance et la première année de maternelle. 


\section{SITOGRAPHIE}

\section{Children crossing borders}

Ce site propose une étude de l'accueil des enfants (de) migrants dans les systèmes d'éducation de la petite enfance dans quatre pays européens et aux États-Unis. Ce projet de recherche, dirigé par Joseph Tobin, rassemble de nombreux chercheurs de chaque pays. L'accent est mis sur l'écoute et la confrontation des voix des parents et des professionnels du préscolaire. http://www.childrencrossingborders.org/.

\section{CIDREE (Consortium of Institutions for Development and Research in Education in Europe)}

Le CIDREE est un groupement d'institutions qui s'occupent de la recherche et de la promotion de l'éducation en Europe, il a été fondé en 1990. Son objectif consiste à améliorer le niveau de l'éducation en Europe. Ses membres souhaitent diffuser les connaissances récentes en matière de développement et de recherche. Dans ce but ils organisent des projets communs et proposent des publications spécialisées disponibles sur ce site. http://www.cidree.org/.

\section{DECET (Diversity in Early Childhood Education and Training)}

Le DECET rassemble un réseau d'organisations européennes avec des objectifs communs sur la valorisation de la diversité dans l'éducation de la petite enfance et la formation. Le réseau vise à promouvoir et étudier les soins aux enfants tout en reconnaissant les multiples identités culturelles des enfants et des familles afin de résoudre efficacement les préjugés et la discrimination. Une base de données bibliographique multilingue est disponible sur ce site. http:// www.decet.org/.

\section{EECERA (European Early Childhood Education Research)}

Le site de l'association EECERA a pour but la promotion, le développement et la diffusion de la recherche en éducation de la petite enfance. En lien avec l'association « le furet, petite enfance et diversité ", il a organisé un congrès annuel dont le thème en 2009 était : diversité des éducations des jeunes enfants. http://www.lefuret.org/19th\%20EECERA/Accueil_19th_ eecera.html

\section{UNICEF/Centre de recherche Innocenti}

Le Centre de recherche Innocenti cherche à contribuer au débat public sur le rôle de l'État dans la petite enfance à travers l'analyse des stratégies nationales de promotion de la qualité dans l'éducation préscolaire. Sur ce site, études, recherches et publications sur la petite enfance sont disponibles. http://www.unicef-irc.org/

\section{Working for inclusion}

Sur ce site, on accède par la "Library » à une série de rapports et documents produits tout au long du programme de recherche "Working for inclusion ». Cette recherche vise à examiner comment améliorer les qualifications et les compétences des professionnels de la petite enfance afin de réduire les inégalités et promouvoir l'accès à l'éducation. Financé par le Programme communautaire pour l'emploi et la solidarité sociale (2007-2013), il est également soutenu par le gouvernement écossais. http://www.knowledge.scot.nhs.uk/wfi/the-programme.aspx/. 